\title{
JOINT OPTIMIZATION OF PRODUCTION PLANNING AND VEHICLE ROUTING PROBLEMS: A REVIEW OF EXISTING STRATEGIES
}

\author{
Marc Reimann $^{1 *}$, Roberto Tavares Neto ${ }^{2}$ and Elisabeth Bogendorfer ${ }^{3 \#}$
}

Received December 31, 2012 / Accepted December 1, 2013

\begin{abstract}
Keen competition and increasingly demanding customers have forced companies to use their resources more efficiently and to integrate production and transportation planning. In the last few years more and more researchers have also focused on this challenging problem by trying to determine the complexity of the individual problems and then developing fast and robust algorithms to solve them. This paper reviews existing literature on integrated production and distribution decisions at the tactical and operational level, where the distribution part is modelled as some variation of the well-known Vehicle Routing Problem (VRP). The focus is thereby on problems that explicitly consider deliveries to multiple clients in a less-than-truckload fashion. In terms of the production decisions we distinguish in our review between tactical and operational production problems by considering lot-sizing/capacity allocation and scheduling models, respectively.
\end{abstract}

Keywords: integrated production and distribution problems, lot-sizing, routing, scheduling.

\section{INTRODUCTION}

Since the mid-1980s integrated production-distribution systems (IPDS) have been the subject of an active research area in Operations Research (OR). According to Sarmiento \& Nagi [45] IPDS are models that jointly optimize decision variables of different production and distribution functions in a single optimization model. The optimization is carried out simultaneously (cf. [45]) and possibly involves additional functions, such as inventory (cf. [21]). The practical needs and recent theoretical results regarding techniques for the integrated planning of production and distribution are highlighted in the specialized literature (cf. [46], [21]).

\footnotetext{
*Corresponding author.

${ }^{1}$ Institute of Production and Operations Management, University of Graz, Universitätsstrasse 15, A-8010 Graz, Austria.

E-mail: marc.reimann@uni-graz.at

${ }^{2}$ Federal University of São Carlos, Rodovia Washington Luis, km 235, São Carlos, SP, Brazil.

E-mail: tavares@dep.ufscar.br

3 JCL AG, Am Terminal 1c, A-8402 Werndorf. E-mail: elisabeth.bogendorfer@jcl-logistics.com

\#This work was initiated, while E. Bogendorfer was doing her MSc dissertation at the University of Graz.
} 
One driving force behind supply chain integration is the fierce competition companies have to face in today's global market. As a consequence, they are forced to utilize their resources efficiently by, among other things, reducing lead times and safety stocks. This reduction of safety stocks is supposed to break up the traditional procedure of developing production and distribution plans separately (i.e., in different departments) and sequentially. Reduced inventory leads to a closer linkage between production and distribution operations, which makes the joint planning inevitable.

Another reason for the necessity of an integrated consideration is the increased customers' pressure on companies to offer individual products quickly, as well as the rising number of companies which have adopted direct-sell e-business models as their way of doing business. Consequently, many of them have implemented make-to-order production, meaning that custom-made products are manufactured and delivered within very short lead times. Due to the fact that these companies can only start producing the products after they have received an order and supply them directly to the customer after their completion, they only have little or no inventory at all. In order to maintain a desired on-time delivery performance at minimum total cost the operations must be jointly scheduled.

Apart from that, the integration is also indispensable in supply chains with time-sensitive products that have a very short life-cycle. Such products cannot be stored but must be supplied to the customers immediately after their production. Examples include perishable goods, industrial adhesive materials, or newspapers and mailing.

As per Chen [22] a joint consideration of production and delivery schedules is also advantageous at the operational level when taking higher level decisions in a supply chain. First of all, supply chain planning can benefit since the results of production-delivery scheduling can be used as estimates for input data that are needed in working out production-distribution plans. In addition, due date or lead time setting decisions, which have a direct effect on customer service, can be made more accurately if the interdependency of order due dates, production schedule, and delivery schedule is considered. In spite of this fact, most of the existing lead time setting models do not involve distribution scheduling decisions.

Due to the closer linkage of production and distribution coordinated planning has become inevitable, which is supported by integrated optimization models in OR. A large body of literature on IPDS can be found at the strategic and tactical planning level. Strategic IPDS concern longterm decisions, such as facility allocation, outsourcing (cf. [32]), plant capacities, and transport channels (cf. [21]). For reviews of these models refer to [28], [43] and [49], for obtaining an insight into the modeling and possible solution methods see, for instance, the works by [6], [34], or [35]. Those at the tactical level relate to models dealing with production, shipping and inventory quantities as well as the duration of the production/distribution cycle and can be found, for instance, in [21] and [45]. The recently published review by Mula et al. [42] especially contains solving methods for tactical and/or operational problems as well as their combination with strategic ones (cf. [42]). In another fairly recent review, Chen [22] has dealt with integrated production and outbound distribution scheduling. Surprisingly, many of the reviewed approaches 
presented by [22] use a rather simplified distribution process of direct shipments, whereas in real industrial applications less-than-truckload settings are far more common than full-truckload settings.

The main aim of this paper is thus to provide a state-of-the-art review of those integrated production-distribution problems which include routing decisions. On the production side we will distinguish between tactical models of lot-sizing or capacity allocation and operational models of production scheduling. The paper's target is to offer an insight into the problems already considered, their interrelations, the solution methods used as well as blank spots identified in the research landscape, and directions for future research.

The remainder of this paper is organized as follows. In the next section the methodology used in the literature research and the classification process is presented. Section 3 gives an overview of the existing models. Concluding comments and directions for future research are given in the final section.

\section{METHODOLOGY}

In this chapter the methodology concerning the literature research as well as the classification of existing and relevant IPDS are introduced.

\subsection{Literature Research}

The search process was conducted in two steps. In the first step all the articles citing the reviews by Chen ([21], [22]) were retrieved. Next, scientific-technical bibliographic databases, including e-journal portals such as EBSCO, Emerald, ScienceDirect, and Springer Link, were searched. In the second step the references listed in the papers found served as a continuous search reference.

The search terms included several versions of production-distribution system, such as productiondistribution model, problem and planning, as well as some alternatives to the term integrated, for example synchronized, coordinated and combined. Moreover, the keywords routing, vehicle routing and VRP were used. Finally, wildcard characters and asterisks were employed to find additional variations. Further restrictions, such as published dates or science(s), were not made.

The papers found during that search were then analyzed with respect to their content and papers not dealing with the actual problem solving of specific IPDS were filtered out. This approach led to 37 papers which are characterized by the fact that the problem studied is described, the optimization model as well as the solving method (i.e., in most of the cases the algorithms) are presented, the experiments introduced and the results evaluated. Each of these papers will be discussed in the main part of the paper below.

Four of these papers were published either in conference proceedings or as a $\mathrm{PhD}$ thesis; for the remaining 33 papers, Table 1 shows the distribution of references according to the journals used in the review. 
Table 1 - Distribution of References.

\begin{tabular}{|l|c|c|}
\hline Distribution of Journals & Number & {$[\%]$} \\
\hline Annals of Operations Research & 1 & 3 \\
Computers and Chemical Engineering & 1 & 3 \\
Computers and Industrial Engineering & 2 & 6 \\
Computers and Operations Research & 4 & 12 \\
European Journal of Operations Research & 8 & 24 \\
Flexible Services and Manufacturing & 1 & 3 \\
IIE Transactions & 2 & 6 \\
INFORMS Journal on Computing & 1 & 3 \\
International Journal of Production Economics & 3 & 9 \\
Journal of Business Logistics & 1 & 3 \\
Journal of Scheduling & 1 & 3 \\
Latin America Applied Research & 1 & 3 \\
Management Science & 1 & 3 \\
Mathematical and Computer Modeling & 1 & 3 \\
Naval Research Logistics & 1 & 3 \\
Operations Research Letters & 1 & 3 \\
Production Planning and Control & 1 & 3 \\
Transportation Science & 2 & 6 \\
\hline Total & 33 & 100 \\
\hline
\end{tabular}

From Table 1 we observe the expected result, i.e., that there is a core of journals including the European Journal of Operations Research and Computers and Operations Research, where a significant portion of this research is published. However, besides this core which does not even account for $50 \%$ of the volume of research in this area there is a wide distribution of articles in journals ranging from engineering to computer science to business. This wide distribution reflects the heterogeneity and the interdisciplinarity of the research area very well. However, it also shows that the area has not yet matured and there is still much need to classify the existing approaches and the blank spots where more research is needed.

\subsection{Classification}

The classification process can be divided into two steps. First, the relevant 37 publications were categorized according to the characteristics of their production processes. Thirteen papers deal with joint lot sizing/capacity allocation + vehicle routing problems, while the remaining 24 papers consider joint production scheduling + vehicle routing problems.

The first problem class links medium-term production decisions (i.e., lasting from two weeks to six months) with the operational VRP, and comprises topics such as production/distribution planning, capacity and inventory allocation as well as safety stock planning (cf. [32]). Typical questions involve the lot-sizing as well as the batching of product deliveries, the clustering of clients and the timing of deliveries over multiple periods (see [21]). Hence, the main decisions 
of this category of problems can be translated as: what and how much (and where, in the case of multi-facility problems) must be produced, when to produce it, how much to deliver to the different customers and which routes to use for serving these customers. Figure 1 shows a generic structure of this category.

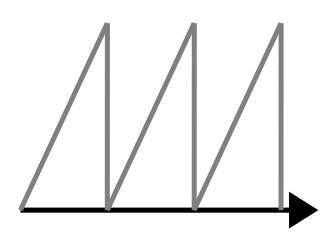

$\rightarrow$ What and how much should be produce?

$\rightarrow$ Where and when production take place?

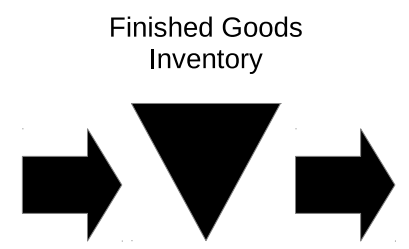

$\rightarrow$ What are the routes of each vehicle?

$\rightarrow$ How much and when should products be delivered?

Figure 1 - A generic structure of a tactical IPDS problem.

The second problem class focuses on short-term decisions (i.e., daily events) and considers detailed production and transportation scheduling problems (cf. [32]). Typical questions involve when and on which machine a job should be processed, when and in which vehicle the products should be delivered and which route each vehicle should choose (see [21]). A generic structure of this category of problems is shown in Figure 2.
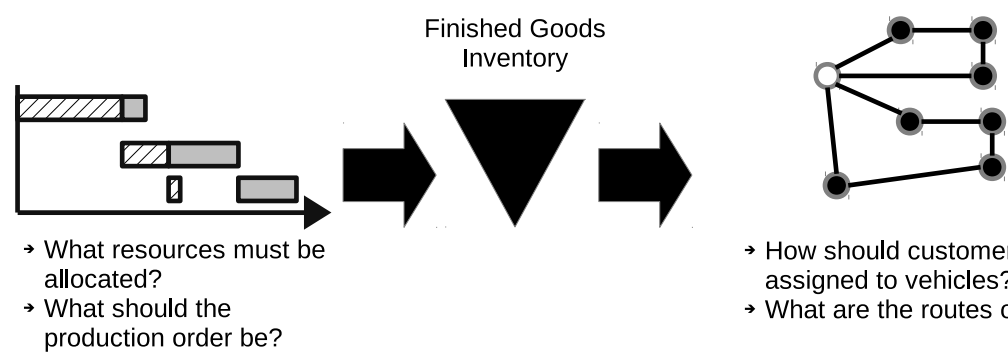

$\rightarrow$ How should customers be assigned to vehicles?

$\rightarrow$ What are the routes of each vehicle?

Figure 2 - A generic structure of an operational IPDS problem.

In the second step, within each class the papers found were then grouped according to the characteristics of the production process and the vehicles, as shown in Table 2. Vehicle characteristics correspond to the fleet size and its composition. On the production side different criteria are used for tactical and operational models. For the former, the number of products and the planning horizon are considered; single product models typically focus on multiple period settings and deal with lot-sizing decisions, while multiple product models relate to capacity allocation settings in a single or multiple periods. In terms of the latter, the scheduling environment can be characterized by a single machine, parallel machines or flowshop setting. Finally, Table 2 also lists the Modeling/Solution approaches that are utilized in the various papers. 
Table 2 - Classification Criteria within each Problem Class.

\begin{tabular}{|c|c|c|}
\hline $\begin{array}{c}\text { Upper Criterion } \\
\text { (Tactical models) }\end{array}$ & Lower Criterion & Alternatives \\
\hline $\begin{array}{c}\text { Time Period(s) } \\
\text { (Tactical models) }\end{array}$ & Single; Multiple \\
\hline $\begin{array}{c}\text { Machine Configuration } \\
\text { (Operational Models) }\end{array}$ & number of vehicles \\
type of fleet & Single; Multiple \\
\hline $\begin{array}{c}\text { Vehicle Characteristics } \\
\text { Modeling/ }\end{array}$ & $\begin{array}{r}\text { Single; Parallel; Flowshop } \\
\text { Solution Approach }\end{array}$ & $\begin{array}{r}\text { One; Limited; Unlimited } \\
\text { Integer Non Linear Programming (INLP); } \\
\text { Lagrangean Relaxation (LR); }\end{array}$ \\
& $\begin{array}{r}\text { Integer Linear Programming ((M)ILP); } \\
\text { Branch \& Bound (B\&B); } \\
\text { (Meta- )Heuristics (HEU) }\end{array}$ \\
\hline
\end{tabular}

During the analysis of each paper, the information about the problem instances used in each paper is also gathered. The results are summarized in Table 3 (to simplify the visualization, only papers with numerical experiments that provided the data used or indicated a publicly available data source are shown).

Table 3 - Information about data sources used in IPDS research.

\begin{tabular}{|l|l|}
\hline \multicolumn{1}{|c|}{ Reference } & \multicolumn{1}{c|}{ Data source } \\
\hline Adulyasak et al. (2012) [1] & \\
Boudia \& Prins (2009) [12] & Modified version of: Solomon MM. Algorithms for the vehicle \\
Boudia et al. (2007) [13] & routing and scheduling problems with time windows constraints. \\
Boudia et al. (2008) [14] & Operations Research,35: 254-265, 1987. \\
Chern \& Hsieh (2007) [24] & Source: https://sites.google.com/site/yossiriadulyasak/publications \\
Lei et al. (2006) [36] & \\
\hline Méndez et al. (2005) [41] & Data provided by the paper \\
\hline
\end{tabular}

\section{REVIEW OF IPDS MODELS INCLUDING ROUTING DECISIONS}

The review consists of two parts and is organized as follows. In the first part tactical problems are treated. The second part of the survey is dedicated to operational problems dealing with joint production scheduling and vehicle routing. Overview tables summarizing the existing results are presented for each class.

\subsection{Tactical IPD problems}

The existing models involve the integration of lot sizing or capacity allocation problems with distribution problems to customers (i.e., end customers, distribution centers, retailers). When 
comparing the papers to be reviewed, the first group deals with an integrated production-inventory-distribution routing problem (PIDRP) determining the production quantities of a single product, the inventory and delivery quantities as well as the routes ([1], [7], [8], [9], [12], [13], [14], and [36]). In contrast to that multi-product capacity allocation problems are studied in the second group of papers ([5], [15], [17], [20], and [30]). In this latter group the paper by Chen et al. [20] stands out in that it is the only approach dealing with a stochastic setting.

Strictly speaking, all of the models in the first group are based, at least to a certain extent, on the work by Lei et al. [36] in dealing with a single-product capacitated lot-sizing problem (CLSP) in their production and an allocation and vehicle routing problem (VRP) in their distribution part. On the other hand, in the second group the model presented in [17] describes a basic model setting which is also expanded in [30]. Below, we show an exemplary formulation of a basic model for tactical IPD problems which combines the features of the models in [36] and [17].

The following notation is used:

Sets:

$H$... set of production facilities

$J$... set of customers/distribution centers (DC)

$K \ldots$ set of products

$P$... set of periods in the planning horizon

$V$... set of vehicles

Indexes:

$h$... production facility index

$j, j^{\prime} \ldots$ customer/DC indexes

$k$... product index

$p$... period index

$v$... vehicle index

Parameters:

$d_{k, j, p} \ldots$ demand of product $k$ at customer $j$ in period $p$

$a_{h, k}^{\text {prod }} \ldots$ per-unit capacity consumption of product $k$ in production facility $h$

$a_{h, k}^{\text {setup }}$... capacity consumption for setup of product $k$ in production facility $h$

$A_{h}^{\text {prod }} \ldots$ total available capacity in production facility $h$

$t_{v, j, j^{\prime}} \ldots$ travel time of vehicle $v$ between customer locations $j$ and $j^{\prime}$

$T_{v} \ldots$ maximum admissible travel time of vehicle $v$

$A_{v}^{\text {vehicle }}$... capacity of vehicle $v$

$I_{k, h}^{\min } \ldots$ safety stock of product $k$ at production facility $h$

$I_{k, j}^{\min } \ldots$ safety stock of product $k$ at customer $j$

$I_{k, h}^{\max } \ldots$ maximum inventory level of product $k$ at production facility $h$

$I_{k, j}^{\max } \ldots$ maximum inventory level of product $k$ at customer $j$

$M$... a sufficiently large number 
$c_{k, h}^{\text {setup }} \ldots$ setup cost for product $k$ at production facility $h$

$c_{k, h}^{p r o d} \ldots$ per-unit production cost for product $k$ at production facility $h$

$c_{k, h}^{i n v-p r o d} \ldots$ per-unit holding cost for product $k$ at production facility $h$

$c_{k, j}^{i n v-c u s t} \ldots$ per-unit holding cost for product $k$ at customer $j$

$c_{v, h}^{v e h i c l e} \ldots$ fixed cost for vehicle $v$ located at production facility $h$

$c_{h, j, k}^{\text {out }} \ldots$ per-unit cost for outsourced transportation of product $k$ delivered from production facil-

ity $h$ to customer $j$

$c_{v, j, j^{\prime}}^{\text {travel }} \ldots$ travel cost of vehicle $v$ travelling directly from $j$ to $j^{\prime}$

Variables:

$x_{k, h, p} \ldots$ production quantity of product $k$ in production facility $h$ in period $p$

$y_{k, h, p} \ldots$ binary decision variable indicating whether or not a setup for product $k$ in production facility $h$ in period $p$ is necessary

$q_{h, j, k, p} \ldots$ shipping quantity of product $k$ from production facility $h$ to customer $j$ in period $p$

$Q_{h, j, k, p} \ldots$ outsourced shipping quantity of product $k$ from production facility $h$ to customer $j$ in period $p$

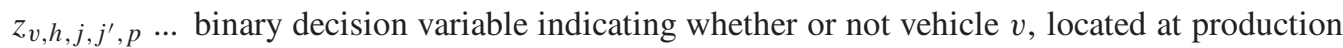
facility $h$ travels directly from $j$ to $j^{\prime}$ in period $p$

$L_{v, h, j, j^{\prime}, p} \ldots$ load of vehicle $v$, located at production facility $h$ when traveling between $j$ and $j^{\prime}$ in period $p$

$$
\begin{aligned}
\min & \sum_{k \in K} \sum_{h \in H} \sum_{p \in P}\left(c_{h, k}^{\text {setup }} \cdot y_{k, h, p}+c_{h, k}^{\text {prod }} \cdot x_{k, h, p}+c_{h, k}^{\text {inv-prod }} \cdot I_{k, h, p}\right) \\
& +\sum_{k \in K} \sum_{h \in H} \sum_{j \in J} \sum_{p \in P} c_{h, j, k}^{\text {out }} \cdot Q_{h, j, k, p} \\
& +\sum_{v \in V} \sum_{h \in H} \sum_{j \in J} \sum_{p \in P} c_{v, h}^{\text {vehicle }} \cdot z_{v, h, h, j, p} \\
& +\sum_{v \in V} \sum_{h \in H} \sum_{j \in\{h\} \cup J} \sum_{j^{\prime} \in\{h\} \cup J} \sum_{p \in P} c_{v, j, j^{\prime}}^{\text {travel }} \cdot z_{v, h, j, j^{\prime}, p} \\
& +\sum_{k \in K} \sum_{j \in J} \sum_{p \in P} c_{h, k}^{\text {inv-cust }} \cdot I_{k, j, p}
\end{aligned}
$$

subject to

$$
\begin{array}{ll}
I_{k, h, p}=I_{k, h, p-1}+x_{k, h, p}-\sum_{j \in J} q_{h, j, k, p}-\sum_{j \in J} Q_{h, j, k, p} & \forall h \in H, k \in K, p \in P \\
I_{k, j, p}=I_{k, j, p-1}-d_{k, j, p}+\sum_{h \in H} q_{h, j, k, p}+\sum_{h \in H} Q_{h, j, k, p} & \forall j \in J, k \in K, p \in P \\
I_{k, h}^{\min } \leq I_{k, h, p} \leq I_{k, h}^{\max } & \forall h \in H, k \in K, p \in P \\
I_{k, j}^{\min } \leq I_{k, j, p} \leq I_{k, j}^{\max } & \forall h \in H, k \in K, p \in P
\end{array}
$$




$$
\begin{aligned}
& \sum_{k \in K}\left(a_{h, k}^{\text {setup }} \cdot y_{k, h, p}+a_{h, k}^{\text {prod }} \cdot x_{k, h, p}\right) \leq A_{h}^{\text {prod }} \quad \forall h \in H, p \in P \\
& x_{k, h, p} \leq M \cdot y_{k, h, p} \quad \forall h \in H, k \in K, p \in P \\
& \sum_{j^{\prime} \in\{h\} \cup J, j^{\prime} \neq j} z_{v, h, j^{\prime}, j, p}=\sum_{j^{\prime \prime} \in\{h\} \cup J, j^{\prime \prime} \neq j} z_{v, h, j, j^{\prime \prime}, p} \quad \forall h \in H, j \in J, v \in V, p \in P \\
& \sum_{j \in J} z_{v, h, h, j, p} \leq 1 \quad \forall h \in H, v \in V, p \in P \\
& \sum_{j \in\{h\} \cup J} \sum_{j^{\prime} \in\{h\} \cup J, j^{\prime} \neq j} t_{v, j, j^{\prime}} \cdot z_{v, h, j, j^{\prime}, p} \leq T_{v} \quad \forall v \in V, p \in P \\
& L_{v, h, j, j^{\prime}, p} \leq A_{v}^{v e h i c l e} \cdot z_{v, h, j, j^{\prime}, p} \quad \forall h \in H, j, j^{\prime} \in J, v \in V, p \in P \\
& \sum_{j^{\prime} \in\{h\} \cup J, j^{\prime} \neq j} L_{v, h, j^{\prime}, j, p}-\sum_{j^{\prime \prime} \in\{h\} \cup J, j^{\prime \prime} \neq j} L_{v, h, j^{\prime \prime}, j, p} \\
& =\sum_{k \in K} q_{h, j, k, p} \quad \forall h \in H, j \in J, v \in V, p \in P \\
& \sum_{j \in J} L_{v, h, j, h, p}-\sum_{j^{\prime} \in J} L_{v, h, h, j^{\prime}, p}=\sum_{k \in K} \sum_{j^{\prime \prime} \in J} q_{h, j^{\prime \prime}, k, p} \quad \forall h \in H, v \in V, p \in P \\
& x_{k, h, p} \geq 0, y_{k, h, p} \in\{0,1\} \\
& \forall h \in H, k \in K, p \in P \\
& q_{h, j, k, p} \geq 0, Q_{h, j, k, p} \geq 0 \\
& \forall h \in H, j \in J, k \in K, p \in P \\
& z_{v, h, j, j^{\prime}, p} \in\{0,1\}, L_{v, h, j, j^{\prime}, p} \geq 0
\end{aligned}
$$

Objective (1) minimizes the total cost consisting of setup, production and inventory cost at the production facilities (first line), cost for outsourced transportation (second line), fixed and variable cost for in-house operated transportation (third line) and inventory cost at the customers (fourth line).

Constraints (2) and (3) are the inventory balance constraints at the production facilities and customers, respectively. Constraints (4) and (5) impose safety stock and maximum inventory levels at the production facilities and customers, respectively. Constraints (6) make sure that the available capacity at each production facility is not violated. The fact that production of a product can only take place when the facility is set up for this product is modelled by constraints (7). Constraints (8) are the flow conservation constraints, implying that a vehicle entering a customer node also needs to leave this customer node. Constraints (9) account for the utilization of vehicles by modelling the first trip of a vehicle leaving its production facility. Constraints (10) make sure that the maximum admissible travel time of any vehicle is not violated. Constraints (11)-(13) account for the vehicle capacity and impose that there can not be any subtours among customer nodes not connected to any production facility. Finally, constraints (14)-(16) set the domains of the decision variables. 
As mentioned above, this model combines features of the models presented in [36] and [17]. While all of the single product models in Table 4 are based on the model by Lei et al. [36] they also feature some important differences. Multiple plants and heterogeneous vehicles are only considered by Lei et al. [36], setup costs are included in all papers except for [36], and different solution approaches are presented. In short, a two-phase methodology is proposed in [36], which means that the routes are determined separately in the second phase of the approach. The model presented and solved in [13] has also been used for the solution methodologies proposed in [1] (adaptive large neighborhood search), [12] (reactive greedy randomized adaptive search procedure) and [14] (memetic algorithm with population management). On the basis of [13] and the two-phase solution approach of [36] Bard \& Nananukul developed a reactive tabu search procedure in [7]. The idea of coupling heuristics with decomposition methods and branch-and-price respectively to efficiently solve the PIDRP is presented in [8] and [9]. More details about these paper are now presented.

Table 4 - Tactical IPDS Problems.

\begin{tabular}{|c|c|c|c|c|c|}
\hline \multirow{2}{*}{$\begin{array}{c}\text { Number of } \\
\text { Products }\end{array}$} & \multirow{2}{*}{$\begin{array}{c}\text { Time } \\
\text { Period(s) }\end{array}$} & \multicolumn{2}{|c|}{ Vehicle Characteristics } & \multirow{2}{*}{$\begin{array}{l}\text { Modelling } \\
\text { Approach }\end{array}$} & \multirow{2}{*}{ References } \\
\hline & & Number & Type & & \\
\hline Single & Multiple & Limited & Heterogeneous & $\begin{array}{l}\text { MIP, HEU } \\
\text { MIP, HEU } \\
\text { MIP, HEU } \\
\text { ILP, HEU } \\
\text { ILP, HEU } \\
\text { ILP, HEU } \\
\text { MIP, HEU } \\
\text { HEU }\end{array}$ & $\begin{array}{c}\text { Bard \& Nananukul (2009a) } \\
\text { Bard \& Nananukul (2009b) } \\
\text { Bard \& Nananukul (2010) } \\
\text { Boudia et al. (2007) } \\
\text { Boudia et al. (2008) } \\
\text { Adulyasak et al. (2012) } \\
\text { Lei et al. (2006) } \\
\text { Boudia \& Prins (2009) }\end{array}$ \\
\hline Multiple & $\begin{array}{l}\text { Single } \\
\text { Multiple }\end{array}$ & $\begin{array}{l}\text { Limited } \\
\text { Limited } \\
\text { Limited, } \\
\text { Unlimited } \\
\text { Unlimited } \\
\text { Limited }\end{array}$ & $\begin{array}{l}\text { Heterogeneous } \\
\text { Homogeneous }\end{array}$ & $\begin{array}{l}\text { MIP } \\
\text { INLP, HEU } \\
\text { MIP, HEU } \\
\text { MIP, HEU } \\
\text { MIP, LR }\end{array}$ & $\begin{array}{c}\text { Aydinel et al. (2008) } \\
\text { Chen et al. (2009) } \\
\text { Bredström \& Rönnqvist (2002) } \\
\text { Chandra \& Fisher (1994) } \\
\text { Fumero \& Vercellis (1999) }\end{array}$ \\
\hline
\end{tabular}

Lei et al. [36] consider a two-stage PIDRP involving multiple production plants and customer demand centers, which both have limited inventory capacities. The production part of the model is characterized by a single-item, single-level CLSP without backlogging and is solved in the first phase of a two-phase solution approach. By contrast, the dissolving of the distribution part comprises both phases. At first, a transportation problem formulated as mixed-integer programming (MIP) model is solved by determining the optimal delivery quantities and trips per transporter. In order to find optimal routes, a delivery consolidation problem formulated in a similar way to the CVRP with the multiple use of vehicles is solved in the second phase. The solution is found by a heuristic transporter routing algorithm based on an extended optimal partitioning procedure. 
Heterogeneous in-house as well as chartered transporters, which can be used for several trips per period, and their traveling times (independent of the quantities delivered) are also incorporated in the model. The objective function minimizes production costs, inventory holding costs of the plants and demand centers, and total transportation costs. The computational performance of the solution approach was tested using 49 randomly generated test problems and a real-life supply network problem of a chemical company shipping products by water transportation. The solutions of the proposed approach were compared to those obtained by the MIP CPLEX solver. It has been shown that in 34 out of 49 cases CPLEX solver either could not find a feasible solution within the given time limit or produced a solution of worse quality than the proposed approach. With respect to the application, 2 plants, 13 demand centres, 3 types of vessel, and 12 time periods were considered. Interplant distribution for the shipment of raw materials and maintenance schedules were also included.

Boudia et al. [13] and Boudia \& Prins [12] developed similar MIP models for the problem described above but considered a single-plant case. Also, setup costs are included in the production part of the models. The distribution parts deals with the CVRP assuming a homogeneous fleet. The aim of the models is the minimization of the sum of setup, inventory holding and transportation costs. They propose a reactive greedy randomized adaptive search procedure (GRASP) in [13] and a memetic algorithm with population management and path-relinking in [12]. In the former work the problem is formulated as an integer linear program and solved by a GRASP metaheuristic which was either improved by a reactive mechanism or a path-relinking process. The algorithm was tested on 90 randomly generated instances with 50, 100, or 200 customers and 20 time periods. Four metaheuristics (basic GRASP, reactive version, interleaved path relinking, and path relinking at the end) were compared with two earlier heuristics, namely a two-phase decoupled approach and a weakly coupled approach (also considered in [14]). It has been shown that the best results are obtained by using GRASP but higher savings are achievable when using the reactive and path relinking version. In [12] several versions of a memetic algorithm with population management were considered and compared with the results obtained either by using GRASP or the sequential two-phase heuristic from [13]. The tests have shown that large-scale problems can be solved within a reasonable time and significant savings are achievable.

In [1] a deterministic single product, single plant, multiple customer problem with limited storage capacity at both the plant and the customers is considered. An adaptive large neighborhood search algorithm (ALNS) is proposed which is based on a problem decomposition approach. For each 200-client instance, different setup schedules are generated for which production quantities and delivery schedules are determined. Each of the solutions (with a different setup schedule) is then improved using the ALNS which considers customer-period combinations and tries to reschedule the shipment to a customer and the routing associated with that shipment. Computational results are presented with regard to the performance relative to some competing algorithms while no analysis is done concerning the integration value of the decisions.

The production part of the model presented in [7] is characterized by a single-plant, single-item CLSP including setup costs and the distribution part comprises a transportation problem and a 
CVRP assuming a homogeneous fleet that deliveries to 50, 100 or 200 clients. A similar solution approach to the one by Lei et al. [36] is presented and tested with three data sets provided by Boudia et al. [13]. The objective function minimizes the sum of production setup costs, a surrogate for the routing costs (fixed and variable costs) as well as inventory holding costs at the plant and customer sites. The researchers propose a reactive tabu search algorithm, using a dynamic tabu list, that comprises two-phases, of which the first is divided into two parts. Part one deals with determining the production and delivery quantities on each day (formulated as a MIP) and part two with finding solutions to the routing problem. In order to achieve these objectives the values obtained in the first part are used as demand data for the CVRP subroutine (based on the tabu search technique) treated in the second part. A neighborhood search is performed in the second phase of the solution approach to improve the current results. For quality reasons, lower bounds are also determined by solving a modified version of the lot-sizing distribution model (phase one). Additionally, path-relinking is used in a post-processing phase to achieve marginal cost reductions. It has been shown that, compared to the results obtained in [13] (by using GRASP), improvements could be achieved but the run time was three to five times longer. The researchers have also concluded that path relinking is not very effective.

In [8] Bard \& Nananukul present the results of coupling heuristics with decomposition methods to find solutions to the 50, 100 and 200 clients-size PIDRP instances examined in [7]. For the computations, a previously developed branch-and-price (B\&P) algorithm is used that requires the solution of multiple inventory routing problems to generate columns for the master problem in each period. In order to improve the results, the researchers developed three heuristics for solving the inventory-routing problem component along with a model for determining periods in which at least one customer requires a delivery. Several experiments were carried out to evaluate the performances of the proposed solution approaches and to find out the most effective algorithm configuration. Computational results obtained by the usage of heuristics with different column generation strategies (adding one or multiple columns in each iteration) at the root node of the $\mathrm{B} \& \mathrm{P}$ search tree as well as incorporating them into the $\mathrm{B} \& \mathrm{P}$ algorithm are provided. Results are also given for solving the problem using CPLEX and the tabu search proposed in [7] or the exact B\&P algorithm.

Bard \& Nananukul's recently published paper [9] provides another similar solution approach for the problem studied in [8]. Methodological contributions of the proposed decomposition algorithm based on B\&P comprise a new branching rule to deal with the degeneracy characteristics of the master problem and a new approach for handling symmetry. Apart from that, a column generation and rounding heuristic were combined to improve the algorithm's efficiency. Extensive testing was carried out to compare the results achieved by the exact B\&P algorithm with those when using the suggested B\&P heuristic with different features. It has been observed that for instances with up to 50 customers and eight time periods the latter approach obtained high quality solutions within one hour and outperfromed both CPLEX and the exact $\mathrm{B} \& \mathrm{P}$ algorithm alone. 
Differing from the models described above, multi-product problems have been studied in [5], [15], [17], [20], and [30]. In contrast to the last three papers, the first two papers deal with real-life problems, multiple production facilities, deterministic demands, and multi-period planning horizon. Besides, they both do not consider delivery time windows. However, they differ in the extent of the production part studied. In [5] only the allocation of up to 500 orders in 16 shipments is considered, while sequencing considerations are included in [15]. In contrast to the other models, the paper by Chen et al. [20] is the only one that deals with stochastic assumptions, a single time period, and delivery time windows. The models and solution methodologies are described in more detail below.

Chandra \& Fisher [17] consider a 2-stage, multi-product problem with a single production facility and multiple customers. Demand for each product in each period is deterministic and has to be satisfied without backlog. There is a setup cost for producing a product in each period. Inventory is allowed at both the plant and the customers. Transportation cost consists of a fixed part and a variable part which is determined by the routes of the vehicles, and hence vehicle routing is one of the decisions of the problem. The problem is formulated as a MIP and the authors compare sequential (first production, then transportation) and integrated approaches to instance sets of up to 10 products and 50 clients. They make the following observations based on computational tests on randomly generated data sets on various parameters: (i) Value of production-distribution coordination (measured as average cost reduction achieved by the coordination) increases with production capacity, vehicle capacity, number of customers, number of products, and number of time periods; (ii) value of coordination increases with relatively high distribution costs (fixed and variable) compared to production cost; and (iii) cost reduction varies from $3 \%$ to $20 \%$. In some cases (e.g. when vehicle capacity is small), there is no value in coordination because in this case all deliveries will be made as full truckloads and hence no consolidation is necessary.

A similar problem is studied by Fumero \& Vercellis ([30]). They additionally assume that there is a limited number of vehicles available for product delivery in each time period. Based on a different MIP formulation they solve the problem using Langrangean relaxation. Also, by comparing coordination with the sequential production-distribution approach they obtain similar results to [17].

Aydinel et al. [5] consider a real-life multi-plant problem of a forest products company which receives orders from multiple customers and ships their products either by train or a truck-railcar combination. Two mixed integer models differing in the assumptions concerning the distribution part are presented. The production part is given by a multi-plant order allocation problem and does not include any lot-sizing in the strict sense. In both models the distribution part comprises the determination of the carriers, the shipment sizes, and the routes. However, the supplier strictly chooses one of the transportation modes (open-mode model). The models were solved by CPLEX and tested using real order files of the company comprising two weeks, of which 309 railcar and 16 truck shipments were considered in the first week followed by 208 railcar and 21 truck shipments in the second. The results were compared to those of the company's current approach and revealed that cost savings ranging from $1.9 \%$ to $2.4 \%$ could be achieved. 
In [15] Bredström \& Rönnqvist study a real-life problem of a Swedish paper mill company consisting of a three-stage supply chain: suppliers of raw materials (logs and wood chips) or imported materials, production sites (mills), and customers (domestic and foreign ones). The researchers provide two mixed-integer-linear-programming (MILP) models, one for the production and one for the distribution problem, but do not actually solve them in an integrated manner. The production plan problem is divided into two subproblems determining the sequence and the products to be produced and the recipe to be used in each time period. The distribution plan model comprises a multi-period, multi-commodity transportation problem and a CVRP with heterogeneous vehicles. It is assumed that the shipments of foreign logs to the production mills are carried out by cargo-ships, the distribution to foreign customers either by cargo-ships hired on a long-term basis or a rented boat for short trips. Deliveries are made by train or truck. A time discretization (weeks, days, periods) is also included. The objective function of the production model includes transportation, storage, and production plan costs, whereas the linear objective function of the distribution covers the sum of flow, storage, and boat usage costs. Concerning the solution approach, column generation together with a heuristic including constrained branching is used in the production plan model, whereas the distribution model is divided into subproblems that are solved repeatedly using $\mathrm{B} \& \mathrm{~B}$ on the integer variables. For the integration of the production and ship scheduling, the researchers propose two methods, either the sequential resolving of the subproblems or a two-phase approach. The latter proposition is similar to the methodology used in [36]. Computational results are not provided.

The last tactical-operational model found in the literature is the one by Chen et al., also a multiproduct model but with stochastic demands (cf. [20]). A two-stage, single-period problem involving a supplier who produces perishable goods and delivers them to multiple retailers within an allowed time window is considered in this paper. The production part of the model is characterized by a single-machine scheduling problem, which determines the production quantities of the products as well as the time of starting the production of the first commodity per vehicle. Shortage costs, delay penalties, and decay rates are also incorporated in the model. A CVRP with soft time windows is given in its distribution part. A mixed integer non linear programming model is formulated with the aim of maximizing the supplier's total profit (i.e., the sum of the expected revenue if the demand is less than the quantity supplied or the expected revenue minus goodwill loss if the demand is higher than the supplied quantity minus production and transportation costs as well as a penalty in case of exceeding the allowed time window for delivery). For the problem solving, the problems are decomposed into two subproblems and solved by the Nelder-Mead method with boundary constraints (first problem) and by a heuristic algorithm (second problem) respectively. Several tests were carried out with the result that the problem can be solved within ten minutes for a maximum of 75 retailers. As opposed to the former solution process, in most cases, it takes a few hours to find a local optimal solution when using LINGO 10.0. Additionally, various sensitivity analyses (determining the correlation between the rate of decay and the supply quantity ratio, between the different time windows and numbers of vehicles, and between the average loading ratio and the number of vehicles) were conducted. The results have 
shown that the fleet size is an important factor leading to a trade-off between deterioration and increased transportation costs.

\subsection{Operational IPD problems}

The problems described in this section involve detailed scheduling of both production and distribution operations. As already stated by Chen [22] only a very limited number of papers in the literature deal with detailed integrated production-distribution scheduling problems. The characteristics of these papers are summarized in Table 5.

Table 5 - Operational IPD Problems.

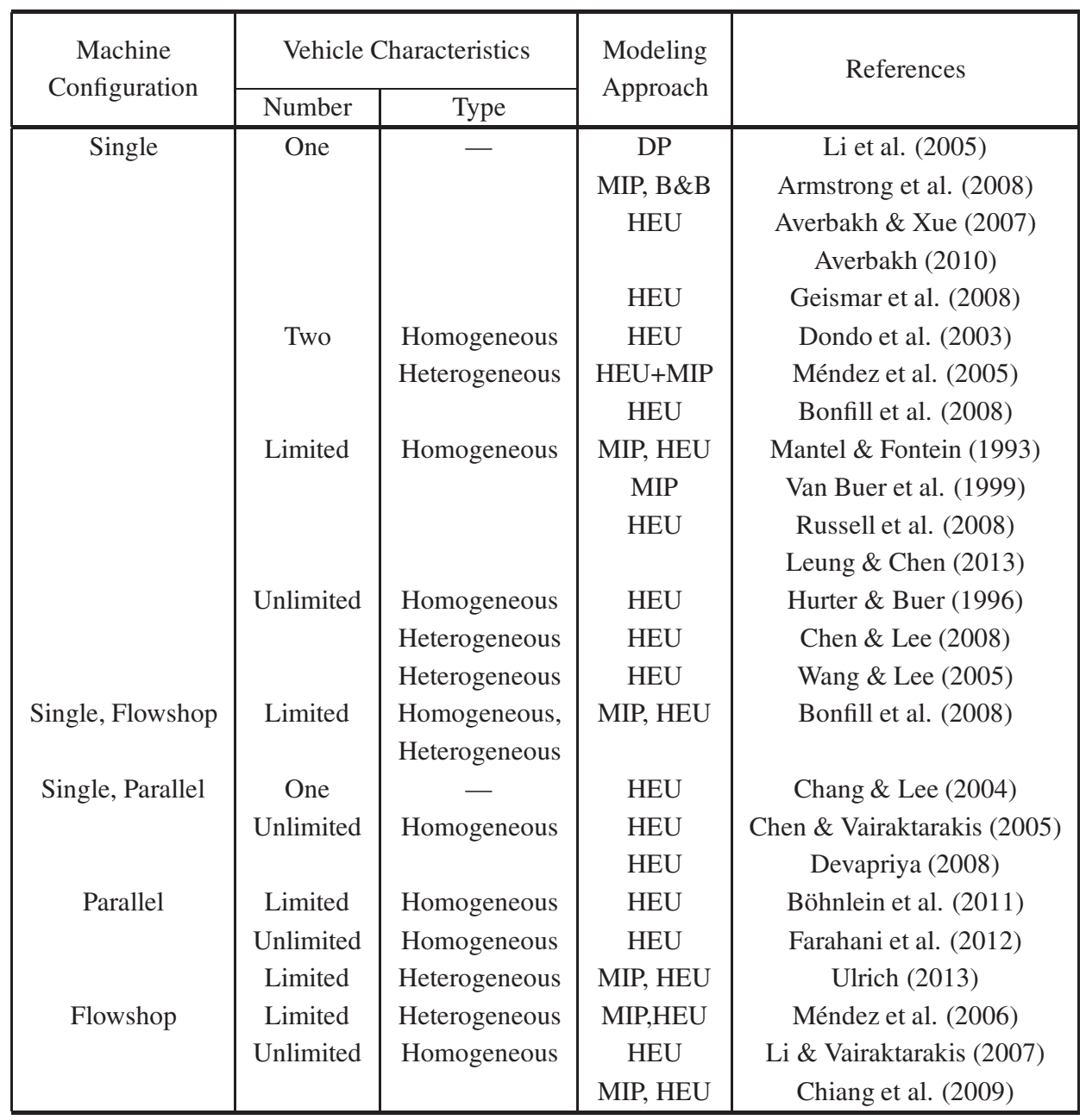

While the explicit models studied in these papers differ significantly due to their operational nature and practical application there are some basic concepts that define integrated production- 
distribution models on the operational level. A recent example nicely highlighting these concepts is the MIP model described by [47]. In this problem, the goal is to minimize the total tardiness of all jobs in an IPD system, where the production site is modeled as a parallel machine environment and the distribution is performed by $t$ routes from $v$ vehicles. The model is based on the following notation:

Sets:

$V$... set of vehicles

$J$... set of customers/jobs, tours

$K$... set of machines

Indexes:

0 ... production facility index

$i, j$... customer/job indexes

$v$... vehicle index

$k$... machine index

$t$... vehicle tour index

Parameters:

$A_{v}^{\text {vehicle }}$... capacity of vehicle $v$

$d_{j} \ldots$ due date of job $j$

$q_{j} \ldots$ size of job $j$

$p_{j} \ldots$ processing time of job $j$

$M$... a sufficiently large number

$r_{k} \ldots$ ready time for machine $k$

$\hat{r}_{v} \ldots$ ready time for vehicle $v$

$s_{0} \ldots$ service time at the production facility

$s_{j} \ldots$ service time at destination of job $j$

$t_{i, j} \ldots$ travel time between delivery sites for jobs $i$ and $j$

$\underline{w}_{j}, \bar{w}_{j} \ldots$ lower and upper bound of the delivery time window of job $j$

Variables:

$C_{j} \ldots$ Completion time of job $j$

$D_{j} \ldots$ Delivery time of job $j$

$S_{v, t} \ldots$ Start time of tour $t$ of vehicle $v$

$T_{j} \ldots$ Tardiness of job $j$

$g_{j, v, t} \ldots$ binary variable that is 1 when job $j$ is delivered by tour $t$ of vehicle $v$

$x_{i, j} \ldots$ binary variable that is 1 when job $j$ is processed after job $i$

$y_{k, j} \ldots$ binary variable that is 1 when job $j$ is the first one to be processed on machine $m$

$z_{i, j, v, t} \ldots$ binary variable that is 1 when job $j$ is delivered after immediately after job $i$ on the tour $t$ of vehicle $v$ 
The first block of constraints (18)-(21) is related to the manufacturing process. The second one is related to the distribution site and shown in constraints (22)-(35). The objective function is presented in equation (17).

$$
\begin{aligned}
& \min \sum_{j \in J} T_{j} \\
& \sum_{j \in J} y_{k, j} \leq 1 \\
& \sum_{k \in K} y_{k, j}+\sum_{i \in J \cup\{0\}, i \neq j} x_{i, j}=1 \\
& C_{j} \geq C_{i}+p_{j}-M \cdot\left(1-x_{i, j}\right) \\
& \forall i, j \in J \\
& C_{j} \geq y_{k, j} \cdot\left(r_{k}+p_{j}\right) \\
& \forall j \in J, k \in K \\
& g_{0, v, t} \geq g_{j, v, t} \\
& \sum_{v \in V} \sum_{t \in J} g_{j, v, t}=1 \\
& M \cdot \sum_{j=1}^{|J|} g_{j, v, t} \geq \sum_{j=1}^{|J|} g_{j, v, t+1} \\
& \forall j \in J, v \in V, t \in J \\
& \forall j \in J \\
& \forall v \in V, t=1, \ldots,|J|-1 \\
& g_{j, v, t}=\sum_{i \in J \cup\{0\}, i \neq j} z_{i, j, v, t} \\
& \forall j \in J, v \in V, t \in J \\
& g_{j, v, t}=\sum_{i \in J \cup\{0\}, i \neq j} z_{j, i, v, t} \\
& \forall j \in J, v \in V, t \in J \\
& A_{v}^{\text {vehicle }} \geq \sum_{j \in J} q_{j} \cdot g_{j, v, t} \\
& \forall v \in V, t \in J \\
& S_{v, 1} \geq \hat{r}_{v}+s_{0} \\
& \forall v \in V \\
& S_{v, t} \geq C_{j}+s_{0}-M \cdot\left(1-g_{j, v, t}\right) \\
& \forall j \in J, v \in V, t \in J \\
& S_{v, t+1} \geq D_{j}+s_{j}+t_{j, 0}+s_{0}-M \cdot\left(1-g_{j, v, t}\right) \\
& \forall j \in J, v \in V, t=1, \ldots,|J|-1 \\
& D_{j} \geq \underline{w}_{j} \\
& \forall j \in J \\
& D_{j} \geq S_{v, t}+t_{0 j}-M \cdot\left(1-g_{j, v, t}\right) \\
& \forall j \in J, v \in V, t \in J \\
& D_{j} \geq D_{i}+s_{i}+t_{i, j}-M \cdot\left(1-z_{i, j, v, t}\right) \\
& \forall i, j \in J, i \neq j, v \in V, t \in J \\
& T_{j} \geq 0 \\
& \forall j \in J \\
& T_{j} \geq D_{j}-\bar{w}_{j}
\end{aligned}
$$


Constraints (18) assure that only one job is the first one to be processed on each machine. Constraints (19) state that a job is sequenced either on the first position of a machine or after some already sequenced job. Constraints (20)-(21) state the boundaries for the completion times of each job on the manufacturing stage.

Constraints (22) state that a tour will include the processing site $(j=0)$ if it performs any delivery. Constraints (23) guarantee that each job is assigned to a unique tour of a single vehicle. Constraints (24) assure that there are no empty tours before an active tour. Constraints (25)-(26) assure that a vehicle that delivers a job $j$ travels from another customer or from the processing site. Constraints (27) limit the vehicles capacity. Constraints (28)-(30) limit the minimum value of the starting time of each route. Constraints (31)-(33) limit the minimum value of the delivery time of each job $j$. The tardiness is bounded by constraints (34)-(35).

As mentioned above this model does not cover all practical characteristics studied in the different papers shown in Table 5. Rather it gives a flavor of some of the main issues that form the basis of operational IPD systems. Below we will now review the specifics of the existing papers in this area.

A practical well known motivation for the study on joint production scheduling/vehicle routing problems is the newspaper production and distribution problem. This problem usually comprehends one or more facilities where different sections of the daily version of the newspaper are printed. Production has to take place within a very short time period (e.g., Russell et al. [44] indicates a scenario where production occurs between midnight and 3 a.m.). Different geographic regions, containing different clients, can compose the daily versions with different sets of sections. The goal is to deliver the different newspaper compositions to the different clients respecting some time window-like constraints (e.g., in the case of the problem studied by [44], the last delivery must occur by 4 a.m.), minimizing some fitness function (such as distribution cost). One possible expansion of this problem is to consider the loading docks as a scarce resource, allowing just a limited number of simultaneous vehicle loadings. This problem is described by Hurter and Buer [33] as the problem of distributing highly perishable products under severe time constraints.

To address the newspaper production and distribution problem, the literature offers some different approaches. The first approach found is the one proposed by Mantel \& Fontein [40], which presents an MILP formulation for the problem, as well as heuristics based on Floyd's method and Clarke \& Wright's method to plan the newspaper deliveries to up to 60 delivery sites. Hurter \& Buer [33] also approach this problem by presenting a two-stage algorithm. A single-vector representation of the problem is proposed by Van Buer et al. [48]. Combined with some definitions of different neighborhoods, a Simulated Annealing and a Tabu Search approach to the problem are proposed. The proposed methods were applied to a dataset generated based on real data originating from a medium-size newspaper company. A similar production system is studied by Chiang et al. [25]. In their case, the problem is characterized by three different daily editions ("class of products"), to be produced in two identical production lines. There are seven distinct delivery zones. To solve this problem, the authors present a mathematical model and a two-phase heuristic approach, that incorporates a reactive tabu search to refine the results obtained by the application 
of an integer programming model in a generalized version of the original problem. To evaluate the effectiveness of the heuristic, Chiang et al. [25] use a simulation model. This simulation model also allows the authors to analyze the effects of the variability on the results provided by the heuristic. A tabu search is also used by Russell et al. [44] to optimize the existing company's operations of an American newspaper company. In this case, there are six different geographic zones supplied by 47 vehicles that deliver to 818 carriers. The carriers cover more than 200 zip codes. The application of the proposed heuristic generates savings of more than $\$ 60,000$ a year. Significant savings on practical scenarios are also presented by Böhnlein et al. [10], who apply a 5-type multi-agent system to real data from one of the largest German newspaper companies, and allow about $17 \%$ of reduction on the variable costs on the planning of the utilization of a 61-vehicle fleet.

Besides newspaper production scenarios, another practical motivation for integrating scheduling and distribution is presented by Geismar et al. [31]. In this case, the authors present a problem encountered in chemical adhesive materials manufacturing. This problem is similar to the newspaper planning problem in the sense that both products (chemical adhesives and newspapers) are products with a relatively short lifespan (hours in the case of newspapers, seven days in the adhesive case presented by [31]). In their approached problem, Geismar et al. present a production system composed of a single plant, a single capacitated vehicle and an uncorrelated set of up to 50 clients dispersed randomly, aiming to minimize the total arrival date of the orders. Although the paper does not present data provided from a specific company, the random-generated instances allow the authors to present some interesting results: a lower bound for the problem considering makespan, as well as genetic and memetic algorithms to solve it. In both cases, the developed heuristic is a 2-stage algorithm designed as follows: in the first stage, a genetic/memetic algorithm generates a sequence of orders; the second stage uses a shortest path algorithm to allocate and sequence the orders in routes. The authors report statistical evidence that, with a $\rho$-value $=0.0005$, the genetic algorithm performed better than the memetic algorithm.

Another practical relevance of integrated production and transportation decisions in the operational level is presented by Farahani et al. [29]. In their paper, the authors analyze how the quality of catering foods can be improved by minimizing the time that the produced orders wait to be shipped. Their claim is that, for this category of food products, the quality of a delivered order decreases according to the time spent in transit after production. The authors develop an iterative solution procedure based on a Large Neighborhood Search algorithm and successfully apply it to 50, 100 and 200-order instances. The results shows that the planning technique proposed allows a better quality of the delivered food without a considerable increase in total costs.

Beyond direct practical applications, one can find in the literature studies that deal with different scenarios of integrated production-transportation problems. A single-machine production stage, now allowing preemption, is approached by Averbakh [3] and Averbakh \& Xue [4]. Starting with a single-client scenario and then moving to a multi-client scenario, both [3] and [4] present a set of different theoretical analyses and algorithms related to the problem of minimizing the sum of total weighted flowtime and delivery costs. 
Chang \& Lee [18] analyze random-generated scenarios by considering a production system where a set of jobs is performed on a single machine or two identical machines, and then grouped in batches for transportation by a single capacitated vehicle to one or two client areas. Some properties of those problems are derived. For the single machine/single client problem, an NPHardness proof is derived. To minimize makespan in a single machine/single vehicle/single vehicle area problem, Chang \& Lee state a property of this problem, where there is an optimal schedule in which: (i) jobs are processed without idle time; (ii) all jobs that are allocated to a vehicle are processed sequentially in the production stage; (iii) there is no order precedence between each production order belonging to the same route; (iv) each distribution batch is delivered according to the well-known Short Processing Time (SPT) rule. This property allows Chang $\&$ Lee to derive a heuristic that uses the well-known First Fit Decreasing (FDD) bin-packing rule for the stated problem. The previous problem is then expanded into a two identical parallel machines production environment, stated as being NP-hard in the strong sense. To solve this problem, a heuristic is also proposed. Another extension presented by the authors is regarding the one machine/one vehicle/two customers area, also stated as NP-Hard in the strong sense. Using results from the FDD rule and the well-known Johnson's rule (used to solve the $F 2 / / C_{\max }$ problem), a heuristic is also proposed. All the proposed heuristics are followed by proofs of worst-case performance.

As with Averbakh \& Xue [4], Chen \& Lee [19] are devoted to presenting theoretical analysis to an integrated production-transportation problem. They approach the problem of producing a set of orders on a single machine. Once produced, the orders must be delivered by a multi-mode (¿2) distribution system. Multiple client sites are considered. The goal is to minimize a weighted function between job delivery time and transportation costs. A multi-mode distribution system is also approached by Wang \& Lee [50]. This latter paper deals with two transportation modes that can be used to transport orders produced by a single machine environment. The due dates are strongly considered in this paper, which presents strategies to: (i) minimize transportation costs in a zero-tardiness solution and (ii) minimize the weighted sum of total tardiness and transportation costs. A B\&B algorithm is presented and, in the instances presented (up to 20 clients), solves the problem more efficiently.

Following this line of research, Méndez et al. [41] consider a comprehensive multi-stage production and transport scheduling problem. The production part of the proposed model deals with identifying the batches to be produced, their assignment to the lines, the sequencing, and the timing. The distribution part provides a delivery schedule comprising the loads, the assignment of orders to the capacitated vehicles, the routes, and the timing of the deliveries. Regarding the transport scheduling, it is assumed that the transport time includes the travel time, a discharge time depending on the amount delivered, and a fixed stop time. Delivery due dates, slack times, and average speeds of the vehicles are also considered. The objective is to minimize weighted total costs including weight values for the travel time, the number of routes, as well as the earliness and tardiness of the orders. In order to reduce the computational effort, different heuristic rules (earliest due date rule, minimum slack time rule, clustering of distribution centres) are 
combined and embedded in the proposed continuous time-based MILP model. A case study is provided assuming that two products are manufactured in a single batch production facility and distributed among eight retail outlets by two vehicles with different capacities. The production recipe for both products comprises three production stages and eight operations. Also, various weight values, ranging from 5 to 100, are included in the objective function. The computational results of the MILP are compared with those of eight hybrid MILP-heuristic approaches, stating that better solutions with modest computational effort can be obtained in the latter case.

The work by Bonfill et al. [11] is based on the paper by Méndez et al. [41], and provides different heuristic-based solution approaches to the problems examined therein. The production scheduling part uses a rule-based heuristic algorithm suggested in [16], whereas in the transport scheduling part an own rule-based heuristic algorithm for the order selection, vehicle assignment, loading, and timing is developed. A two-stage sequential approach (with the production part being solved first) and an integrated strategy (i.e., production orders and due dates are updated in accordance, containing temporal requirements implied by the transport schedule) are provided. Several versions of the proposed solving methods with different combinations of the priority rules are tested in two case studies. The first is very similar to the case study presented in [41] and is solved by the integrated approach with three criteria (i.e., minimum summed lateness, minimum flow time, and minimum multiple cost). Due to its structure, the second problem, an adapted version of the problem treated in [27], is tested by the sequential approach only. This study comprises a single-product, single-stage facility problem, in which the finished product has to be delivered to ten destinations by two homogeneous vehicles. The results have also been compared to those achieved by the approach used in [41]. The researchers have concluded that the application of the presented integrated algorithm leads to better performances in various aspects (e.g., material flow management).

Chen \& Vairaktarakis [23] also approached a similar type of problem. In this case, the manufacturing system is composed of a production phase - stated as a single machine or a set of identical parallel machines - and a distribution phase - composed of one or multiple capacitated vehicles that deliver products to multiple (up to 160) clients geographically dispersed. Inspired by real-world applications such as adhesive chemicals manufacturing, a zero-inventory policy between the production and the distribution phases is stated. The objective function is composed of a weighted sum of the service level and the distribution cost. The customer service level is measured by the maximum delivery time or by the average delivery time. Those definitions - two manufacturing environments, two fleet specifications (single and multiple vehicles) and two different definitions of the objective functions - generate a set of eight different problems. Exact algorithms are stated for the following cases: single machine, one or multiple vehicles, minimizing average distribution time and total distribution costs; parallel machine, one vehicle, minimizing average distribution time and total distribution costs. The remaining problems are solved by heuristics proposed in the paper. A comparison of the solutions obtained by the integrated approach and sequential approaches for the problems is then performed. The authors realize that, for problems with an objective function composed of the average delivery time, the 
integrated approach generates a gain of $5 \%$ on the fitness value. It is also realized that when the number of clients increases, this difference tends to increase. When analyzing problems that minimize the maximum delivery time, the gain is more than $5 \%$ for problems with more than three vehicles and where the weight factor of the objective function emphasizes the delivery time.

Armstrong et al. [2] consider an integrated problem composed of a single machine that produces a product with a limited lifespan. The production is delivered by a single capacitated vehicle under a fixed client delivery sequence subject to a time windows constraint. This problem is stated as NP-Hard. The authors present an MIP model, as well as a heuristic to determine a lower bound for the problem. Furthermore, a B\&B procedure is presented to obtain the optimal value. According to the results presented by Armstrong et al., the time required to solve the 100 clientsproblem using the $\mathrm{B} \& \mathrm{~B}$ approach was significantly lower than the time required by a commercial solver to solve the MIP model: in the best case, the time of the B\&B approach was close to $1.5 \%$ of the commercial solver time; in larger instances - e.g., with 50 customers - the MIP model could not be solved, but the B\&B approach found the result in less than two minutes.

Li et al. [39], approach a single manufacturing facility with distribution accomplished by a set of one or infinite bounded capacity vehicles to a set of one or multiple clients. The goal is to minimize the total arrival time of the orders. The authors prove that this is an NP-Hard problem in the strong sense for the multiple clients case, and $O\left(n^{2}\right)$ for the single client case. To solve this problem, the authors derive a dynamic programming approach that shows a complexity of a polynomial function of the number of clients when the number of clients is greater than one, and with lower complexity if the vehicle is uncapacitated.

A similar problem is approached by Devapriya [26]. The author approaches two different sets of problems: a single plant problem (solved by three different heuristics) and a multi-plant problem (solved by five heuristics). Sets of 40, 60 and 80 clients are considered. When several production facilities are considered, the vehicle fleet of each facility is independent, and a vehicle is not allowed to visit more than one production site. The developed heuristics are based on the concept of first applying a routing algorithm and then a cluster algorithm.

Two different approaches for the transportation problem were used by Li \& Vairaktarakis [38]. In their specific case, the production stage is represented as a 2-machine flowshop environment, that produces up to 80 orders. The distribution phase is carried by a third party carrier, with an unlimited number of vehicles. The orders are delivered to up to five different locations, where each location contains several customers. The objective is to minimize the sum of the client waiting costs and transportation costs. In this specific problem, the transportation is modelled as direct deliveries or as a milk-run. To solve those problems, the authors develop a polynomialtime approximation scheme for the problem. Further heuristics with guaranteed lower bounds are also developed.

A bi-objective planning of a single-machine production site and a capacitated vehicle transportation system is presented by Leung \& Chen [37]. In their paper, the problem of minimiz- 
ing maximum lateness is extended to determine an algorithm that allows one, given a minimum/maximum lateness, to obtain the minimum fleet size. Moreover, an algorithm to minimize the weighted sum of the maximum lateness and the number of used vehicles is presented. Although no numerical examples are presented, proofs of optimality and complexity of the proposed algorithm are discussed in the paper.

\section{FINAL REMARKS AND POTENTIAL FOR FUTURE RESEARCH}

The objective of this paper was to present a review of integrated production and distribution planning models which include routing decisions. In order to provide a structured and clear survey, the research results have been classified according to their decision level, into tactical and operational problems.

In conclusion, the integrated planning of production and distribution operations is critical in today's business. In order to achieve optimal performance, mathematical optimization models might deliver decisive information if the functions are integrated and jointly planned. Such models of IPDS including routing aspects have gained an increasing research interest especially in the last few years. However, the research results show that a wide range of problems remain open for future research. Concerning the transportation part of the model, aspects such as delivery time windows, split deliveries or backhauls could be considered. With respect to the production part, the study of multi-machine and multi-product problems or combinations of tactical and operational problems is suggested. Apart from that, models including multiple production sites and stochastic assumptions need to be investigated to a larger extent. With regard to the solution approaches faster and more robust algorithms need to be developed to treat complex IPDS arising in real-world applications.

\section{ACKNOWLEDGMENTS}

Roberto F. Tavares Neto thanks FAPESP/Brazil for its financial support (process 2011/14800-3).

\section{REFERENCES}

[1] Adulyasak Y, Cordeau J-F \& Jans R. 2012. Optimization-based adaptive large neighborhood search for the production routing problem. Transportation Science.

[2] Armstrong R, Gao S \& Lei L. 2008. A zero-inventory production and distribution problem with a fixed customer sequence. Annals of Operations Research, 159(1): 395-414.

[3] AverbaKh I. 2010. On-line integrated production-distribution scheduling problems with capacitated deliveries. European Journal of Operational Research, 200: 377-384.

[4] Averbakh I \& XUE Z. 2007. On-line supply chain scheduling problems with preemption. European Journal of Operational Research, 181: 500-504.

[5] Aydinel M, Sowlati T, Cerda X, Cope E \& Gerschman M. 2008. Optimization of production allocation and transportation of customer orders for a leading forest products company. Mathematical and Computer Modelling, 48: 1158-1169. 
[6] BARbarosoǦLu G \& ÖZGÜR D. 1999. Hierarchical design of an integrated production and 2echelon distribution system. European Journal of Operational Research, 118(3): 464-484.

[7] BARD JF \& NANANUKUL N. 2009. The integrated production-inventory-distribution-routing problem. Journal of Scheduling, 12: 257-280.

[8] BARD JF \& NANANUKUL N. 2009. Heuristics for a multiperiod inventory routing problem with production decisions. Computers \& Industrial Engineering, 57: 713-723.

[9] BARD JF \& NANANUKUL N. 2010. A branch-and-price algorithm for an integrated production and inventory routing problem. Computers \& Operations Research, 37: 2202-2217.

[10] Böhnlein D, Schweiger K \& Tuma A. 2011. Multi-agent-based transport planning in the newspaper industry. International Journal of Production Economics, 131: 146-157.

[11] Bonfill A, Espuña A \& PUigJaner L. 2008. Decision support framework for coordinated production and transport scheduling in scm. Computers \& Chemical Engineering, 32: 1206-1224.

[12] Boudia M \& PRins C. 2009. A memetic algorithm with dynamic population management for an integrated production-distribution problem. European Journal of Operational Research, 195: $703-$ 715.

[13] Boudia M, Louly MAO \& Prins C. 2007. A reactive grasp and path relinking for a combined production-distribution problem. Computers \& Operations Research, 34: 3402-3419.

[14] Boudia M, Louly MAO \& Prins C. 2008. Fast heuristics for a combined production planning and vehicle routing problem. Production Planning \& Control, 19(2): 85-96.

[15] BREDSTRÖM D \& RÖNNQVIST M. 2002. Integrated production planning and route scheduling in pulp mill industry. In HICSS '02: Proceedings of the 35th Annual Hawaii International Conference on System Sciences (HICSS'02)-Volume 3, Washington, DC, 2002. IEEE Computer Society.

[16] CANTON J. 2003. Integrated support system for planning and scheduling of batch chemical plants. $\mathrm{PhD}$ thesis, Universitat Politecnia de Catalunya.

[17] Chandra P \& Fisher ML. 2004. Coordination of production and distribution planning. European Journal of Operational Research, 72: 503-517.

[18] Chang Y-C \& LeE C-Y. 2004. Machine scheduling with job delivery coordination. European Journal of Operational Research, 158(1): 470-487.

[19] CHEN B \& LEE C-Y. 2008. Logistics scheduling with batching and transportation. European Journal of Operational Research, 189: 871-876.

[20] Chen H-K, Hsueh C-F \& Chang M-S. 2009. Production scheduling and vehicle routing with time windows for perishable food products. Computers \& Operations Research, 36: 2311-2319.

[21] Chen Z-L. 2004. Integrated production and distribution operations: Taxonomy, models, and review. In: D. Simchi-Levi, S.D. Wu, and Z.-J. Shen, editors, Handbook of Quantitative Supply Chain Analysis: Modeling in the E-Business Era. Kluwer Academic Publishers.

[22] Chen Z-L. 2010. Integrated production and outbound distribution scheduling: Review and extensions. Operations Research, 58(1): 130-148.

[23] Chen Z-L \& VAIRAKTARAKIS GL. 2005. Integrated scheduling of production and distribution operations. Management Science, 51(4): 614-628. 
[24] CHERn C-C \& HsieH J-S. 2007. A heuristic algorithm for master planning that satisfies multiple objectives. Computers \& Operations Research, 34: 3491-3513.

[25] Chiang W, Russell R, Xu X \& Zepeda D. 2009. A simulation/metaheuristic approach to newspaper production and distribution supply chain problems. International Journal of Production Economics, 121: 752-767.

[26] DeVAPRIYA P. 2008. Optimal feet size of an integrated production and distribution scheduling problem for a single perishable product. $\mathrm{PhD}$ thesis, Clemson University.

[27] Dondo R, MÉndez CA \& CERdÁ J. 2003. An optimal approach to the multiple-depot heterogeneous vehicle routing problem with time window and capacity constraints. Latin American Applied Research, 33: 129-134.

[28] EREngÜÇ SS, Simpson NC \& VAKharia AJ. 1999. Integrated production/distribution planning in supply chains: An invited review. European Journal of Operational Research, 115: 219-236.

[29] Farahani P, Grunow M \& GÜNTher H. 2012. Integrated production and distribution planning for perishable food products. Flexible Services and Manufacturing, 24: 28-51.

[30] Fumero F \& VerCelLis C. 1999. Synchronized development of production, inventory, and distribution schedules. Transportation Science, 33: 330-340.

[31] Geismar HN, Laporte G, Lei L \& SRiskandarajah C. 2008. The integrated production and transportation scheduling problem for a product with a short life span and non-instantaneous transportation time. INFORMS Journal on Computing, 20(1): 21-33.

[32] HUANG GQ, LAU JSK \& MAK KL. 2003. The impacts of sharing production information on supply chain dynamics: a review of the literature. International Journal of Production Research, 41(7): $1483-1517$.

[33] HuRTER AP \& VAN BUeR MG. 1996. The newspaper productiondistribution problem. Journal of Business Logistics, 17(1): 85-107.

[34] Jayaraman V \& Pirkul H. 2001. Planning and coordination of production and distribution facilities for multiple commodities. European Journal of Operational Research, 133(2): 394-408.

[35] KesKIn BB \& ÜsteR H. 2007. Meta-heuristic approaches with memory and evolution for a multiproduct production/distribution system design problem. European Journal of Operational Research, 182: $663-682$.

[36] Lei L, LiU S, Ruszczynski A \& PARK S. 2006. On the integrated production, inventory, and distribution routing problem. IIE Transactions, 38: 955-970.

[37] LEUnG JYT \& CHEn Z-L. 2013. Integrated production and distribution with fixed delivery departure dates. Operations Research Letters, 41: 290-293.

[38] Li C-L \& VAIRAKTARAKis GL. 2007. Coordinating production and distribution of jobs with bundling operations. IIE Transactions, 39(2): 203-215.

[39] Li C-L, Vairaktarakis GL \& Lee C-Y. 2005. Machine scheduling with deliveries to multiple customer locations. European Journal of Operational Research, 164(1): 39-51.

[40] MANTEL RJ \& FonteIN M. 1993. A practical solution to a newspaper distribution problem. International Journal of Production Economics, 30: 591-599. 
[41] Méndez C, Bonfill A, Espuña A \& Puigjaner L. 2006. A rigorous approach to coordinate production and transport scheduling in a multi-site system. In: MARQUARDT W \& PANTELIDES C, editors, 16th European Symposium on Computer Aided Process Engineering and 9th International Symposium on Process Systems Engineering, volume 21 of Computer Aided Chemical Engineering, pages 2171-2176. Elsevier.

[42] Mula J, Peidro D, Díaz-Madroñero M \& Vicens E. 2010. Mathematical programming models for supply chain production and transport planning. European Journal of Operational Research, 204: $377-390$.

[43] OWEN SH \& DASKIN MS. 1998. Strategic facility location: A review. European Journal of Operational Research, 111(3): 423-447.

[44] Russell R, Chiang W \& Zepeda D. 2008. Integrating multi-product production and distribution in newspaper logistics. Computers \& Operations Research, 35: 1578-1588.

[45] Sarmiento AM \& NAgi R. 1999. A review of integrated analysis of production-distribution systems. Technical report, Department of Industrial Engineering, State University of New York and Buffalo, Buffalo, NY 14260.

[46] Stecke KE \& ZhaO X. 2007. Production and transportation integration for a make-to-order manufacturing company with a commit-to-delivery business mode. Manufacturing \& Service Operations Management, 9: 206-224.

[47] ULRICH CA. 2013. Integrated machine scheduling and vehicle routing with time windows. European Journal of Operational Research, 227: 152-165.

[48] VAN Buer MG, WoodrufF DL \& Olson RT. 1999. Solving the medium newspaper production/distribution problem. European Journal of Operational Research, 115: 237-253.

[49] Vidal CJ \& Goetschalckx M. 1997. Strategic production-distribution models: A critical review with emphasis on global supply chain models. European Journal of Operational Research, 98: 1-18.

[50] WANG H \& LeE C-Y. 2005. Production and transport logistics scheduling with two transport mode choices. Naval Research Logistics, 52: 796-809. 